\title{
Jones-matrix Formalism as a Representation of the Lorentz Group
}

\author{
D. Han* \\ National Aeronautics and Space Administration, Goddard Space Flight Center, Code 910.1, \\ Greenbelt, Maryland 207ry1 \\ Y. S. Kim ${ }^{\dagger}$ \\ Department of Physics, University of Maryland, College Park, Maryland 20742 \\ Marilyn E. Noz $\ddagger$ \\ Department of Radiology, New York University, New York, New York 10016
}

\begin{abstract}
It is shown that the two-by-two Jones-matrix formalism for polarization optics is a six-parameter two-by-two representation of the Lorentz group. The attenuation and phase-shift filters are represented respectively by the threeparameter rotation subgroup and the three-parameter Lorentz group for two spatial and one time dimensions. It is noted that the Lorentz group has another three-parameter subgroup which is like the two-dimensional Euclidean group. Possible optical filters having this Euclidean symmetry are discussed in detail. It is shown also that the Jones-matrix formalism can be extended to some of the non-orthogonal polarization coordinate systems within the framework of the Lorentz-group representation.
\end{abstract}

\footnotetext{
*electronic mail: han@trmm.gsfc.nasa.gov

†electronic mail: kim@umdhep.umd.edu

‡electronic mail: noz@nucmed.med.nyu.edu
} 


\section{INTRODUCTION}

The Lorentz group was introduced to physics as the language of space-time symmetries of relativistic particles [1, 2]. However, this group serves useful purposes in many other branches of physics, including optical sciences. In recent years, the Lorentz group served as the underlying language for squeezed states of light. It was Dirac who first observed that the Lorentz boost is a squeeze transformation [3]. In 1963 [4], Dirac constructed representations of the Lorentz group using coupled harmonic oscillators. Indeed, Dirac's oscillator representation forms the theoretical foundations of squeezed states of light [5] 7 . This aspect of the Lorentz group is by now well known in the optics community, and the Lorentz group is one of the theoretical tools in optics.

The squeezed state is not the only branch of optics requiring the Lorentz group. In 1981, Bacry and Cadihilac considered application of the Lorentz group for Fourier optics [8]. In 1983, Sudarshan et al. constructed a representation of the Lorentz group for para-axial wave optics [9]. Wavelets are known to be representations of the Lorentz group [10]. It has been noted recently that the Lorentz group serves as the underlying language for reflections and

refractions [11]. In addition, in their recent paper [12], Gutierrez et al. pointed out the relevance of the Lorentz geometry to three-dimensional concentrators.

More recently, the relevance of Lorentz transformations to polarization optics has been discussed by several authors [11,13 16]. Indeed, in their recent paper [16, Opartrny and Perina have shown that rotation and boost matrices from the two-by-two representation of the Lorentz group describe energy-conserving and non-conserving optical filters respectively. In our recent paper, we pointed out that polarization optics can be formulated in terms of the six-parameter Lorentz group [17].

The purpose of this paper is very simple. The standard language for optical polarizations is the Jones-matrix formalism, and it has a long history [18 20]. In this paper, we show that this formalism is a representation of the six-parameter Lorentz group. The Lorentz group also has a long history. 
Among the many different representations of this group, as has been shown in one of our earlier papers [17], the bilinear conformal representation [2] is most convenient for polarization optics. Unfortunately, however, we were not able to connect there the representation with the Jones-matrix formalism, and we shall complete the task in the present paper.

The six-parameter Lorentz group has three major subgroups. They are the threeparameter rotation group, the three-parameter $O(2,1)$ group, and the three-parameter $E(2)$ like group. The mathematics of the rotation group is well known. It is shown in Ref. [17] that the rotation group is the underlying language for optical filters causing phase shifts between the two transverse components. The $O(2,1)$-like groups have been extensively discussed recently in connection with squeezed states of light, and it is a very familiar language in optics. It is known that this $O(2,1)$-like group is the proper language for attenuation filters [15, 17].

The E(2)-like group is useful in understanding the internal space-time symmetries of massless particles [21], but is relatively new in optics [22]. We discuss in this paper possible optical filters possessing this $E(2)$-like symmetry. Since this requires a detailed knowledge of the six-parameter Lorentz group, and since this group is relatively new in optics, we shall include in this paper a systematic introduction to the Lorentz group applicable to three spatial and one time-like dimensions.

The rotation and squeeze transformations discussed in this paper are directly applicable to coordinate transformations. Thus, they are applicable to the case of where the polarization occurs along a skew or squeezed coordinate system. It is known that one of the $E(2)$-like transformation leads to a "shear" transformation. The formalism is therefore is applicable also to the case where the polarization coordinate is sheared. The polarization plane is not always perpendicular to the propagation direction of the light wave. The formalism can be extended to accommodate this case.

In Sec. II, we prove that the Jones-matrix formalism is indeed a representation of the Lorentz group. In Sec. III, the mathematics of Sec. [I] is translated into transformation matrices corresponding to optical filters. It is shown that the combined effect of attenuation 
and phase-shift filters leads to a six-parameter two-by-two matrix. It is noted in Sec. [V] that the bilinear conformal representation of the Lorentz group is the natural scientific language for polarization optics. It shown that the attenuation and phase-shift filters have their own sub-representations. It is shown that one of those sub-representations leads to a new kind of optical filter having the $E(2)$-like symmetry. In Sec. $\nabla$, we discuss a possible new class of optical filters having the symmetry of the two-dimensional Euclidean group, and their possible applications in Sec. VI. In Sec. VII, we show that the formalism developed in this paper can accommodate the cases where the polarization coordinates are squeezed or sheared. The formalism can be extended also the case where the polarization plane is not perpendicular to the direction of propagation.

Since the Lorentz group is relatively new in optical sciences, we present the four-by-four and two-by-two representations of this group in Appendices A and B respectively. Since $E(2)$ group plays the central role in this paper, we give an introduction to this group in Appendix C.

\section{FORMULATION OF THE PROBLEM}

In studying polarized light propagating along the $z$ direction, the traditional approach is to consider the $x$ and $y$ components of the electric fields. Their amplitude ratio and the phase difference determine the state of polarization. Thus, we can change the polarization either by adjusting the amplitudes, by changing the relative phases, or both. For convenience,

we call the optical device which changes amplitudes an "attenuator" and the device which changes the relative phase a "phase shifter."

Let us write the electric field vector as

$$
\begin{aligned}
& E_{x}=A \cos \left(k z-\omega t+\phi_{1}\right), \\
& E_{y}=B \cos \left(k z-\omega t+\phi_{2}\right),
\end{aligned}
$$

where $A$ and $B$ are the amplitudes which are real and positive numbers, and $\phi_{1}$ and $\phi_{2}$ are 
the phases of the $x$ and $y$ components respectively. This form is useful not only in classical optics but also applicable to coherent and squeezed states of light [7]23].

The traditional language for this two-component light is the Jones-matrix formalism which is discussed in standard optics textbooks [20]. In this formalism, the above two components are combined into one column matrix with the exponential form for the sinusoidal function.

$$
\left(\begin{array}{c}
E_{x} \\
E_{y}
\end{array}\right)=\left(\begin{array}{c}
A \exp \left\{i\left(k z-\omega t+\phi_{1}\right)\right\} \\
B \exp \left\{i\left(k z-\omega t+\phi_{2}\right)\right\}
\end{array}\right) .
$$

This column matrix is called the Jones vector. The content of polarization is determined by the ratio:

$$
\frac{E_{y}}{E_{x}}=\left(\frac{B}{A}\right) e^{i\left(\phi_{2}-\phi_{1}\right)} .
$$

which can be written as one complex number:

$$
w=r e^{i \phi}
$$

with

$$
r=\frac{B}{A}, \quad \phi=\phi_{2}-\phi_{1} .
$$

The degree of polarization is measured by these two real numbers, which are the amplitude ratio and the phase difference respectively. The purpose of this paper is to discuss the transformation properties of this complex number $w$. The transformation takes place when the light beam goes through an optical filter whose transmission properties are not isotropic.

The text-book version of the Jones-matrix formalism [20] starts with the projection operator:

$$
\left(\begin{array}{ll}
1 & 0 \\
0 & 0
\end{array}\right),
$$

applicable to the Jones vector of Eq.(2.2). This operator keeps the $x$ component and completely eliminates the $y$-component of the electric field. This is an oversimplification of the real world where the attenuation factor in the $y$ direction is greater than that along the $x$ 
direction. We shall replace this projection operator by an attenuation matrix which is closer to the real world.

Another element in the traditional formalism is

$$
P(0, \delta)=\left(\begin{array}{cc}
e^{-i \delta / 2} & 0 \\
0 & e^{i \delta / 2}
\end{array}\right)
$$

which leads to a phase difference of $\delta$ between the $x$ and $y$ components. The polarization axes are not always the $x$ and $y$ axes. For this reason, we need the rotation matrix

$$
R(\theta)=\left(\begin{array}{cc}
\cos (\theta / 2) & -\sin (\theta / 2) \\
\sin (\theta / 2) & \cos (\theta / 2)
\end{array}\right) .
$$

The traditional Jones-matrix formalism consists of systematic combinations of the above three components given in Eq.(2.5), Eq.(2.6) and Eq.(2.7).

In this paper, we replace the projection operator of Eq.(2.5) by a squeeze matrix. There are two transverse directions which are perpendicular to each other. The absorption coefficient in one transverse direction could be different from the coefficient along the other direction. Thus, there is the "polarization" coordinate in which the absorption can be described by

$$
\left(\begin{array}{cc}
e^{-\eta_{1}} & 0 \\
0 & e^{-\eta_{2}}
\end{array}\right)=e^{-\left(\eta_{1}+\eta_{2}\right) / 2}\left(\begin{array}{cc}
e^{\eta / 2} & 0 \\
0 & e^{-\eta / 2}
\end{array}\right)
$$

with $\eta=\eta_{2}-\eta_{1}$. Let us look at the projection operator of Eq.(2.5). Physically, it means that the absorption coefficient along the $y$ direction is much larger than along the $x$ direction. The absorption matrix in Eq.(2.8) becomes the projection matrix if $\eta_{1}$ is very close to zero and $\eta_{2}$ becomes infinitely large. The projection operator of Eq.(2.5) is therefore a special case of the above attenuation matrix.

The attenuation matrix of Eq.(2.8) tells us that the electric fields are attenuated at two different rates. The exponential factor $e^{-\left(\eta_{1}+\eta_{2}\right) / 2}$ reduces both components at the same rate and does not affect the state of polarization. The effect of polarization is solely determined by the squeeze matrix

$$
S(0, \eta)=\left(\begin{array}{cc}
e^{\eta / 2} & 0 \\
0 & e^{-\eta / 2}
\end{array}\right)
$$


This type of mathematical operation is quite familiar from studies of squeezed states of light, if not from Lorentz boosts of spinors. For convenience, we call the above matrix an attenuator. Thus, we are expanding the Jones-matrix formalism by replacing the projection operator of Eq.(2.5) by the squeeze operator in Eq.(2.9).

The phase-shifter of Eq.(2.6) can be written as

$$
P(0, \delta)=\exp \left(-i \delta J_{1}\right)
$$

with

$$
J_{1}=\frac{1}{2}\left(\begin{array}{cc}
1 & 0 \\
0 & -1
\end{array}\right) \text {. }
$$

Our notation for the Pauli sigma matrices is different from those appearing in the conventional literature, and is explained in detail in Appendix D.

The rotation operator of Eq.(2.7) takes the form:

$$
R(\theta)=\exp \left(-i \theta J_{3}\right)
$$

with

$$
J_{3}=\frac{1}{2}\left(\begin{array}{cc}
0 & -i \\
i & 0
\end{array}\right) .
$$

The squeeze operator of Eq.(2.9) can also be written in the exponential form:

$$
S(0, \eta)=\exp \left(-i \eta K_{1}\right)
$$

with

$$
K_{1}=\frac{i}{2}\left(\begin{array}{cc}
1 & 0 \\
0 & -1
\end{array}\right) .
$$

It is now possible to construct a closed set of commutation relations with the above generators $J_{1}, J_{3}$, and $K_{1}$. Then the result is a set of six generators given in Appendix B. They are the generators for the group $S L(2, c)$ which is locally isomorphic to the sixparameter Lorentz group. This is the mathematical content of this paper. Let us next exploit the physical contents of this mathematical formalism. 


\section{COMBINED EFFECTS}

If the polarization coordinate is the same as the $x y$ coordinate where the electric field components take the form of Eq.(2.1), the above attenuator is directly applicable to the column matrix of Eq.(2.2). If the polarization coordinate is rotated by an angle $\theta / 2$, or by the matrix

$$
R(\theta)=\left(\begin{array}{cc}
\cos (\theta / 2) & -\sin (\theta / 2) \\
\sin (\theta / 2) & \cos (\theta / 2)
\end{array}\right),
$$

then the squeeze matrix becomes

$$
\begin{aligned}
S(\theta, \eta) & =R(\theta) S(0, \eta) R(-\theta) \\
& =\left(\begin{array}{ll}
e^{\eta / 2} \cos ^{2}(\theta / 2)+e^{-\eta / 2} \sin ^{2}(\theta / 2) & \left(e^{\eta / 2}-e^{-\eta / 2}\right) \cos (\theta / 2) \sin (\theta / 2) \\
\left(e^{\eta / 2}-e^{-\eta / 2}\right) \cos (\theta / 2) \sin (\theta / 2) & e^{-\eta / 2} \cos ^{2}(\theta / 2)+e^{\eta / 2} \sin ^{2}(\theta / 2)
\end{array}\right) .
\end{aligned}
$$

We can obtain the inverse of this transformation by rotating the filter or the above expression around the $z$-axis by 90 degrees.

If we apply two squeeze matrices, the net result becomes

$$
S\left(\theta_{2}, \eta_{2}\right) S\left(\theta_{1}, \eta_{1}\right)=S\left(\theta_{3}, \eta_{3}\right) R(\psi),
$$

where $R(\psi)$ is a rotation around the $z$ axis by $\psi$. This means that the multiplication of two squeeze matrices does not lead to another squeeze matrix, but a squeeze matrix preceded by a rotation matrix. This aspect of the squeeze operation is well known from the squeezed state of light, and has been discussed extensively in the literature [0,14, 15]. As was noted in Sec. [1], the generators $K_{1}$ and $J_{3}$ are needed for the squeeze matrix of Eq.(3.2). The repeated application leads to the commutation relation for these two generators:

$$
\left[J_{3}, K_{1}\right]=i K_{2} \text {. }
$$

Indeed, $J_{3}, K_{1}$ and $K_{2}$ form a closed set of commutation relations for the $S p(2)$ or $O(2,1)$-like

subgroup of $S L(2, c)$ [15]. This three-parameter subgroup has been extensively discussed in connection with squeezed states of light [0,, 7$]$. 
Another basic element is the optical filter with two different values of the index of refraction along the two orthogonal directions. The effect on this filter can be written as

$$
\left(\begin{array}{cc}
e^{i \delta_{1}} & 0 \\
0 & e^{i \delta_{2}}
\end{array}\right)=e^{-i\left(\delta_{1}+\delta_{2}\right) / 2}\left(\begin{array}{cc}
e^{-i \delta / 2} & 0 \\
0 & e^{i \delta / 2}
\end{array}\right)
$$

with $\delta=\delta_{2}-\delta_{1}$. In measurement processes, the overall phase factor $e^{-i\left(\delta_{1}+\delta_{2}\right) / 2}$ cannot be detected, and can therefore be deleted. The polarization effect of the filter is solely determined by the matrix

$$
P(0, \delta)=\left(\begin{array}{cc}
e^{-i \delta / 2} & 0 \\
0 & e^{i \delta / 2}
\end{array}\right) .
$$

This form was noted as one of the basic components of the Jones-matrix formalism in Sec. [1]. This phase-shifter matrix appears like a rotation matrix around the $z$ axis in the theory of rotation groups, but it plays a different role in this paper. We shall hereafter call this matrix a phase shifter.

Here also, if the polarization coordinate makes an angle $\theta$ with the $x y$ coordinate system, the phase shifter takes the form

$$
\begin{aligned}
P(\theta, \delta) & =R(\theta) P(0, \delta) R(-\theta) \\
& =\left(\begin{array}{ll}
e^{-i \delta / 2} \cos ^{2}(\theta / 2)+e^{i \delta / 2} \sin ^{2}(\theta / 2) & \left(e^{-i \delta / 2}-e^{i \delta / 2}\right) \cos (\theta / 2) \sin (\theta / 2) \\
\left(e^{-i \delta / 2}-e^{i \delta / 2}\right) \cos (\theta / 2) \sin (\theta / 2) & e^{i \delta / 2} \cos ^{2}(\theta / 2)+e^{-i \delta / 2} \sin ^{2}(\theta / 2)
\end{array}\right) .
\end{aligned}
$$

Here again, we can obtain the inverse of this transformation by rotating the filter around the $z$-axis by 90 degrees.

If we consider only the phase shifters, the mathematics is basically repeated applications of $J_{1}$ and $J_{2}$, resulting in applications also of $J_{3}$, where their explicit two-by-two matrix forms are given in Appendix B. Thus, the phase-shift filters form an $S U(2)$ or $O(3)$-like subgroup of the group $S L(2, c)$.

If we use both the attenuators and phase shifters, the result is the full $S L(2, c)$ group with six parameters. The transformation matrix is usually written as

$$
L=\left(\begin{array}{ll}
\alpha & \beta \\
\gamma & \rho
\end{array}\right)
$$


with the condition that its determinant be one: $\alpha \rho-\gamma \beta=1$. The repeated application of two matrices of this kind results in

$$
\begin{aligned}
& \left(\begin{array}{cc}
\alpha_{2} & \beta_{2} \\
\gamma_{2} & \rho_{2}
\end{array}\right)\left(\begin{array}{cc}
\alpha_{1} & \beta_{1} \\
\gamma_{1} & \rho_{1}
\end{array}\right) \\
& =\left(\begin{array}{cc}
\alpha_{2} \alpha_{1}+\beta_{2} \gamma_{1} & \alpha_{2} \beta_{1}+\beta_{2} \rho_{1} \\
\gamma_{2} \alpha_{1}+\rho_{2} \gamma_{1} & \gamma_{2} \beta_{1}+\rho_{2} \rho_{1}
\end{array}\right)
\end{aligned}
$$

The most general form of the polarization transformation is the application of this algebra to the column matrix of Eq.(2.2).

The generators of the rotation or phase-shifters are Hermitian. Thus, the unitary subset

of the $L$ matrices of Eq.(3.8), it represents the three-parameter rotation-like subgroup or the phase-shifters and their repeated applications. The generators $J_{3}, K_{1}$ and $K_{2}$ are all imaginary. Thus, the real subset of the $L$ matrices represents the attenuation filters and their repeated applications.

\section{BILINEAR CONFORMAL REPRESENTATION OF THE LORENTZ GROUP}

In the present formulation of polarization optics, we are interested in calculating one complex variable defined in Eq.(2.4). As was noted in Ref. [17], we can obtain the same algebraic result by using the bilinear transformation :

$$
w^{\prime}=\frac{\rho w+\gamma}{\beta w+\alpha}
$$

The repeated applications of these two transformations can be achieved from

$$
w_{1}=\frac{\rho_{1} w+\gamma_{1}}{\beta_{1} w+\alpha_{1}}, \quad w_{2}=\frac{\rho_{2} w_{1}+\gamma_{2}}{\beta_{2} w_{1}+\alpha_{2}}
$$

Then, it is possible to write $w_{2}$ as a function of $w$, and the result is

$$
w_{2}=\frac{\left(\gamma_{2} \beta_{1}+\rho_{2} \rho_{1}\right) w+\left(\gamma_{2} \alpha_{1}+\rho_{2} \gamma_{1}\right)}{\left(\alpha_{2} \beta_{1}+\beta_{2} \rho_{1}\right) w+\left(\alpha_{2} \alpha_{1}+\beta_{2} \gamma_{1}\right)}
$$

This is a reproduction of the algebra given in the matrix multiplication of Eq.(3.9). The form given in Eq.(4.1) is the bilinear representation of the Lorentz group [2]. 
Let us consider the physical interpretation of this result. If we apply the matrix $L$ of Eq.(3.8) to the column vector of Eq.(2.2), then

$$
\left(\begin{array}{ll}
\alpha & \beta \\
\gamma & \rho
\end{array}\right)\left(\begin{array}{c}
E_{x} \\
E_{y}
\end{array}\right)=\left(\begin{array}{c}
\alpha E_{x}+\beta E_{y} \\
\gamma E_{x}+\rho E_{y}
\end{array}\right),
$$

which gives

$$
\frac{E_{y}^{\prime}}{E_{x}^{\prime}}=\frac{\gamma E_{x}+\rho E_{y}}{\alpha E_{x}+\beta E_{y}} .
$$

In term of the physical quantity $w$ defined in Eq.(2.4), this formula becomes

$$
w^{\prime}=\frac{\gamma+\rho w}{\alpha+\beta w} .
$$

This equation is identical to the bilinear form given in Eq.(4.1), and the ratio $w$ can now be identified with the $w$ variable defined as the parameter of the bilinear representation of the Lorentz group in the same equation. Indeed, the bilinear representation is clearly the natural language for polarization optics.

Let us next consider subgroups. For the phase-shifters, the subgroup is represented by the unitary matrix

$$
\left(\begin{array}{cc}
\alpha & \beta \\
-\beta^{*} & \alpha^{*}
\end{array}\right) .
$$

This form is preserved in the bilinear representation. As for the attenuation subgroup, all the components are real, and this aspect is also preserved in the bilinear representation.

Let us next consider another form of bilinear transformation.

$$
w^{\prime}=\frac{(\exp (-i \delta / 2) w}{\exp (i \delta / 2)+\beta w} .
$$

This is also a form-preserving transformation. This form can be transformed into the matrix form

$$
\left(\begin{array}{cc}
e^{-i \delta / 2} & \beta \\
0 & e^{i \delta / 2}
\end{array}\right) .
$$

Let us study this sub-representation in detail in Sec. D. 


\section{NEW FILTERS}

We should note at this point that the Lorentz group has another set of three-parameter subgroups. They are like the two-dimensional Euclidean group. Let us consider one of them, which is generated by the matrices $J_{1}, N_{2}$ and $N_{3}$, with

$$
N_{2}=J_{2}+K_{3}, \quad N_{3}=J_{3}-K_{2},
$$

where

$$
N_{2}=\left(\begin{array}{ll}
0 & 1 \\
0 & 0
\end{array}\right), \quad N_{3}=\left(\begin{array}{cc}
0 & -i \\
0 & 0
\end{array}\right) .
$$

These matrices satisfy the commutation relations:

$$
\left[J_{1}, N_{2}\right]=i N_{3}, \quad\left[J_{1}, N_{3}\right]=-i N_{2} \quad\left[N_{2}, N_{3}\right]=0
$$

They indeed form a closed set of commutation relations. As shown in Appendix C, these commutation relations are like those for the two-dimensional Euclidean group consisting of two translations and one rotation around the origin. This group has been studied extensively in connection with the space-time symmetries of massless particles, where $J_{1}$ and the two $N$ generators correspond to the helicity and gauge degrees of freedom respectively 21.

The physics of $J_{1}$ is well known through the phase shifter given in Eq.(2.6). If the angle $\delta$ is $\pi / 2$, the phase shifter becomes a quarter-wave shifter, which we write as

$$
Q=P(0, \pi / 2)=\left(\begin{array}{cc}
e^{-i \pi / 4} & 0 \\
0 & e^{i \pi / 4}
\end{array}\right)
$$

Then $J_{2}$ and $K_{3}$ are the quarter-wave conjugates of $J_{3}$ and $K_{2}$ respectively:

$$
J_{2}=Q J_{3} Q^{-1}, \quad K_{3}=-Q K_{2} Q^{-1}
$$

Consequently,

$$
N_{2}=Q N_{3} Q^{-1}
$$

The $N$ generators lead to the following transformation matrices. 


$$
\begin{aligned}
& T_{2}(\tau)=\exp \left(-i \tau N_{2}\right)=\left(\begin{array}{ll}
1 & i \tau \\
0 & 1
\end{array}\right), \\
& T_{3}(\tau)=\exp \left(-i \tau N_{3}\right)=\left(\begin{array}{cc}
1 & -\tau \\
0 & 1
\end{array}\right) .
\end{aligned}
$$

It is clear that $T_{2}$ is the quarter-wave conjugate of $T_{3}$. We can now concentrate on the transformation matrix $T_{3}$.

If $T_{3}$ is applied to the column matrix of Eq.(2.2),

$$
\left(\begin{array}{cc}
1 & -\tau \\
0 & 1
\end{array}\right)\left(\begin{array}{c}
E_{x} \\
E_{y}
\end{array}\right)=\left(\begin{array}{c}
E_{x}-\tau E_{y} \\
E_{y}
\end{array}\right) .
$$

This new filter superposes the $y$ component of the electric field to the $x$ component with an appropriate constant, but it leaves the $y$ component invariant.

Let us examine how this is achieved. The generator $N_{3}$ consists of $J_{3}$ which generates rotations around the $z$ axis, and $K_{2}$ which generates a squeeze along the $45^{\circ}$ axis. Physically, $J_{3}$ generates optical activities. Thus, the new filter consists of a suitable combination of these two operations. In both cases, we have to take into account the overall attenuation factor. This can be measured by the attenuation of the $y$ component which is not affected by the symmetry operation of Eq.(5.8).

Is it possible to produce optical filters of this kind? Starting from an optically active material, we can introduce an asymmetry in absorption to it by either mechanical or electrical means. Another approach would be to pile up alternately the $J_{3}$-type and $K_{2}$-type layers. In either case, it is interesting to note that the combination of these two effects produces a special effect predicted from the Lorentz group.

The $E(2)$-like symmetry includes transformations generated by $J_{1}$, which is given in Eq.(2.11). The transformation matrix is

$$
P(0, \delta)=\left(\begin{array}{cc}
e^{-i \delta / 2} & 0 \\
0 & e^{i \delta / 2}
\end{array}\right),
$$

This matrix is given in Eq.(2.6) and its physics are well understood. Let us apply this matrix to $T_{3}$ from left and from right. Then 


$$
\begin{aligned}
& P(0, \delta) T_{3}(\tau)=\left(\begin{array}{cc}
1 & -e^{-i \delta / 2} \tau \\
0 & 1
\end{array}\right), \\
& T_{3}(\tau) P(0, \delta)=\left(\begin{array}{cc}
1 & -e^{i \delta / 2} \tau \\
0 & 1
\end{array}\right) .
\end{aligned}
$$

This leads to

$$
\begin{aligned}
P(0,-\delta) T_{3}(\tau) P(0, \delta) & =T_{3}\left(e^{i \delta} \tau\right) \\
& =\left(\begin{array}{cc}
1 & -e^{i \delta} \tau \\
0 & 1
\end{array}\right) .
\end{aligned}
$$

We can of course obtain the $P(0,-\delta)$ filter by rotating the $P(0, \delta)$ around the $z$ axis by 90 degrees. It is thus possible to add a phase factor to the $\tau$ variable using phase shifters of the type $P(0, \delta)$.

\section{POSSIBLE APPLICATIONS OF THE NEW FILTER}

The three-dimensional rotation group occupies an important place in many different branches of physics. The group $O(2,1)$ also is useful in a number of fields including optics [7,24]26]. Thus, traditional attenuation and phase-shift filters may be useful in constructing analog computers performing the symmetry operations of these groups.

The group $E(2)$ is somewhat new in optics [22]. However, as was noted in Appendix C, it deals with translations and rotations on a flat surface. This filter may therefore be useful as a computational device for recording and reading two-dimensional maps. Furthermore, the $T_{3}$ matrix of Eq.(5.7) has an interesting algebraic property:

$$
\left(\begin{array}{cc}
1 & \beta_{1} \\
0 & 1
\end{array}\right)\left(\begin{array}{cc}
1 & \beta_{2} \\
0 & 1
\end{array}\right)=\left(\begin{array}{cc}
1 & \beta_{1}+\beta_{2} \\
0 & 1
\end{array}\right) .
$$

The matrix can therefore be used for converting multiplication into addition, like the logarithmic function. This is one of the most basic operations in computational machines.

As for a more immediate application, let us consider lens optics. It is a trivial laboratory operation to rotate a given filter around the $z$ axis by 90 degrees. If we rotate the matrix of Eq.(6.3), the result is the matrix of the form 


$$
\left(\begin{array}{ll}
1 & 0 \\
\beta & 1
\end{array}\right)
$$

This form together with the original form of Eq. (6.3) serve as lens and translation matrices respectively in para-axial optics. Indeed, a system of polarization filters can serve as an analog computer for a multi-lens system.

Furthermore, the matrix of the form

$$
\left(\begin{array}{ll}
1 & \beta \\
0 & 1
\end{array}\right)
$$

represents a "shear" transformation. This is one of the basic deformations in engineering applications.

The Lorentz group was introduced to physics as the basic language for space-time symmetries of elementary particles [1], but it is becoming increasingly prominent in many branches of physics and engineering including classical and quantum optics. The optical filters may provide excellent calculational tools for the Lorentz group. Thus, these filters may be useful as components of future computers.

\section{NON-ORTHOGONAL COORDINATE SYSTEMS}

Since the light polarization is caused by anisotropic crystals, the polarization coordinate is not always orthogonal. Let us consider first the case where the light polarization is along a pair of skew or squeezed axes.

It was noted in this paper that the $O(2,1)$-like subgroup can take care of attenuation filters. A transformation matrix of this subgroup can also transform the orthogonal coordinate system into a squeezed coordinate system. The matrix takes the form

$$
\left(\begin{array}{l}
x^{\prime} \\
y^{\prime}
\end{array}\right)=\left(\begin{array}{cc}
\cosh (\eta / 2) & \sinh (\eta / 2) \\
\sinh (\eta / 2) & \cosh (\eta / 2)
\end{array}\right)\left(\begin{array}{l}
x \\
y
\end{array}\right) .
$$

The idea is to transform the squeezed coordinate system into the orthogonal system using the matrix or its inverse given in the above expression. Next, we can perform the polarization 
algebra developed in this paper for the orthogonal coordinate system. We then can transform the result obtained in the orthogonal system back to the original squeezed coordinate system.

It is interesting to note that the transformation matrix given in Eq.(7.1) is one of the transformation matrices within the frame work of the Lorentz-group representation developed in this paper, and there is no need to make the existing mathematics more complicated. The story is the same for the shear transformation which takes the form

$$
\left(\begin{array}{l}
x^{\prime} \\
y^{\prime}
\end{array}\right)=\left(\begin{array}{ll}
1 & b \\
0 & 1
\end{array}\right)\left(\begin{array}{l}
x \\
y
\end{array}\right) .
$$

This transformation is also well within the framework discussed in this paper.

The polarization plane is not always perpendicular to the direction of the propagation. We can take care of this problem by extending our two-by-two formalism of Eq.(3.8) into the three-by-three form

$$
\left(\begin{array}{lll}
\alpha & \beta & 0 \\
\gamma & \rho & 0 \\
0 & 0 & 1
\end{array}\right)
$$

applicable to the $(x, y, z)$ coordinate system. The polarization plane can be rotated around the $x$ axis by

$$
\left(\begin{array}{ccc}
1 & 0 & 0 \\
0 & \cos \xi & -\sin \xi \\
0 & \sin \xi & \cos \xi
\end{array}\right) .
$$

Using this matrix or its inverse, we can bring the problem to the orthogonal coordinate system. After doing the standard polarization algebra, we can go back to the original coordinate system.

\section{CONCLUDING REMARKS}

In this paper, we have replaced the projection operator of Eq.(2.5) by the squeeze matrix given in Eq.(2.9)). This brings the Jones-matrix formalism closer to the real world. This also enables us to formulate the problem within the framework of the Lorentz group. 
There are now many powerful mathematical tools derivable from the Lorentz group [7,26]. We can use them to study more systematically varous aspects of optics. The present paper is the first step toward a systematic exposition of polarization optics. There are several interesting future problems. First, it is now possible to study the relation between the Jones vectors and the Stokes parameters using the mathematical theorems of the Lorentz group connecting Minkowskian four-vectors and $S L(2, c)$ spinors. Furthermore, the natural language of the Lorentz group is based on circularly polarized states of photons. It is therefore an interesting future problem to work out the Jones-matrix formalism applicable to circularly polarized lights.

It is widely understood that the Lorentz group started gaining its prominence in optics through squeezed states of light [5]. This is not true. This group was discussed much earlier in connection with polarization optics [31]. However, we learned the connection between squeeze transformations and Lorentz boosts while studying squeezed states of light [7]. The Lorentz group is now a powerful mathematical device because there are many squeeze transformations in physics, including the Jones-matrix formalism discussed in the present paper.

It is also gratifying to note that the symmetry of the Lorentz group is useful for various engineering applications of light waves including their polarizations, reflections, and their propagation media [32]. In physics, it is relatively new to study Maxwell's equations in terms of the Lorentz group [26]. It is thus a challenging future problem to combine the symmetries of electromagnetic waves developed in engineering with those developed in physics.

\section{APPENDIX A: LORENTZ TRANSFORMATIONS}

Let us consider the space-time coordinates $(x, y, z, t)$. Then the rotation around the $z$ axis is performed by the four-by-four matrix 


$$
\left(\begin{array}{cccc}
\cos \theta & -\sin \theta & 0 & 0 \\
\sin \theta & \cos \theta & 0 & 0 \\
0 & 0 & 1 & 0 \\
0 & 0 & 0 & 1
\end{array}\right)
$$

This transformation is generated by

$$
J_{3}=\left(\begin{array}{cccc}
0 & -i & 0 & 0 \\
i & 0 & 0 & 0 \\
0 & 0 & 0 & 0 \\
0 & 0 & 0 & 0
\end{array}\right) .
$$

Likewise we can write down the generators of rotations $J_{1}$ and $J_{2}$ around the $x$ and $y$ axes respectively. These three generators satisfy the closed set of commutations relations

$$
\left[J_{i}, J_{j}\right]=i \epsilon_{i j k} J_{k} .
$$

This set of commutation relations is for the three-dimensional rotation group.

The Lorentz boost along the $z$ axis takes the form

$$
\left(\begin{array}{cccc}
\cosh \eta & 0 & 0 & \sinh \eta \\
0 & 1 & 0 & 0 \\
0 & 0 & 1 & 0 \\
\sinh \eta & 0 & 0 & \cosh \eta
\end{array}\right)
$$

which is generated by

$$
K_{3}=\left(\begin{array}{cccc}
0 & 0 & 0 & i \\
0 & 0 & 0 & 0 \\
0 & 0 & 0 & 0 \\
i & 0 & 0 & 0
\end{array}\right) .
$$

Likewise, we can write generators of boosts $K_{1}$ and $K_{2}$ along the $x$ and $y$ axes respectively, and they take the form

$$
K_{1}=\left(\begin{array}{llll}
0 & i & 0 & 0 \\
i & 0 & 0 & 0 \\
0 & 0 & 0 & 0 \\
0 & 0 & 0 & 0
\end{array}\right), \quad K_{2}=\left(\begin{array}{cccc}
0 & 0 & i & 0 \\
0 & 0 & 0 & 0 \\
i & 0 & 0 & 0 \\
0 & 0 & 0 & 0
\end{array}\right) .
$$


These boost generators satisfy the commutation relations

$$
\left[J_{i}, K_{j}\right]=i \epsilon_{i j k} K_{k}, \quad\left[K_{i}, K_{j}\right]=-i \epsilon_{i j k} J_{k}
$$

Indeed, the three rotation generators and the three boost generators satisfy the closed set of commutation relations given in Eq. A3) and Eq.(A7). These three commutation relations form the starting point of the Lorentz group. The generators given in this Appendix are four-by-four matrices, but they are not the only set satisfying the commutation relations. We can construct also six two-by-two matrices satisfying the same set of commutation relations. The group of transformations constructed from these two-by-matrices is often called $S L(2, c)$ or the two-dimensional representation of the Lorentz group. Throughout the present paper, we used the two-by-two transformation matrices constructed from the generators of the $S L(2, c)$ group.

\section{APPENDIX B: SPINORS AND FOUR-VECTORS IN THE LORENTZ GROUP}

In Appendix A, we have noted that there are four-by-four and two-by-two representations of the Lorentz group. The four-by-four representation is applicable to covariant four-vectors, while the two-by-two transformation matrices are applicable to two-component spinors which

in the present case are Jones vectors. The question then is whether we can construct the four-vector from the spinors. In the language of polarization optics, the question is whether it is possible to construct the coherency matrix [28,29 from the Jones vector.

With this point in mind, let us start from the following form of the Pauli spin matrices.

$$
\begin{aligned}
\sigma_{1} & =\left(\begin{array}{cc}
1 & 0 \\
0 & -1
\end{array}\right), \quad \sigma_{2}=\left(\begin{array}{ll}
0 & 1 \\
1 & 0
\end{array}\right), \\
\sigma_{3} & =\left(\begin{array}{cc}
0 & -i \\
i & 0
\end{array}\right) .
\end{aligned}
$$

These matrices are written in a different convention. Here $\sigma_{3}$ is imaginary, while $\sigma_{2}$ is imaginary in the traditional notation. Also in this convention, we can construct three rotation generators 


$$
J_{i}=\frac{1}{2} \sigma_{i}
$$

which satisfy the closed set of commutation relations

$$
\left[J_{i}, J_{j}\right]=i \epsilon_{i j k} J_{k}
$$

We can also construct three boost generators

$$
K_{i}=\frac{i}{2} \sigma_{i}
$$

which satisfy the commutation relations

$$
\left[K_{i}, K_{j}\right]=-i \epsilon_{i j k} J_{k}
$$

The $K_{i}$ matrices alone do not form a closed set of commutation relations, and the rotation generators $J_{i}$ are needed to form a closed set:

$$
\left[J_{i}, K_{j}\right]=i \epsilon_{i j k} K_{k}
$$

The six matrices $J_{i}$ and $K_{i}$ form a closed set of commutation relations, and they are like the generators of the Lorentz group applicable to the $(3+1)$-dimensional Minkowski space. The group generated by the above six matrices is called $S L(2, c)$ consisting of all two-by-two complex matrices with unit determinant.

In order to construct four-vectors, we need two different spinor representations of the Lorentz group. Let us go to the commutation relations for the generators given in Eqs.(B3), (B5) and (B6). These commutators are not invariant under the sign change of the rotation generators $J_{i}$, but are invariant under the sign change of the squeeze operators $K_{i}$. Thus, to each spinor representation, there is another representation with the squeeze generators with opposite sign. This allows us to construct another representation with the generators:

$$
\dot{J}_{i}=\frac{1}{2} \sigma_{i}, \quad \dot{K}_{i}=\frac{-i}{2} \sigma_{i}
$$

We call this representation the "dotted" representation. If we write the transformation matrix $L$ of Eq.(3.8) in terms of the generators as 


$$
L=\exp \left\{-\frac{i}{2} \sum_{i=1}^{3}\left(\theta_{i} \sigma_{i}+i \eta_{i} \sigma_{i}\right)\right\}
$$

then the transformation matrix in the dotted representation becomes

$$
\dot{L}=\exp \left\{-\frac{i}{2} \sum_{i=1}^{3}\left(\theta_{i} \sigma_{i}-i \eta_{i} \sigma_{i}\right)\right\} .
$$

In both of the above matrices, Hermitian conjugation changes the direction of rotation. However, it does not change the direction of boosts. We can achieve this only by interchanging $L$ to $\dot{L}$, and we shall call this the "dot" conjugation.

Likewise, there are two different set of spinors. Let us use $u$ and $v$ for the up and down spinors for "undotted" representation. Then $\dot{u}$ and $\dot{v}$ are for the dotted representation. The four-vectors are then constructed as 27]

$$
\begin{aligned}
& u \dot{u}=-(x-i y), \quad v \dot{v}=(x+i y) \\
& u \dot{v}=(t+z), \quad v \dot{u}=-(t-z)
\end{aligned}
$$

leading to the matrix

$$
C=\left(\begin{array}{cc}
u \dot{v} & -u \dot{u} \\
v \dot{v} & -v \dot{u}
\end{array}\right)=\left(\begin{array}{l}
u \\
v
\end{array}\right)\left(\begin{array}{ll}
\dot{v} & -\dot{u}
\end{array}\right),
$$

where $u$ and $\dot{u}$ are one if the spin is up, and are zero if the spin is down, while $v$ and $\dot{v}$ are zero and one for the spin-up and spin-down cases. The transformation matrix applicable to the column vector in the above expression is the two-by-two matrix given in Eq.(3.8). What is then the transformation matrix applicable to the row vector $(\dot{v},-\dot{u})$ from the right-hand side? It is the transpose of the matrix applicable to the column vector $(\dot{v},-\dot{u})$. We can obtain this column vector from

$$
\left(\begin{array}{c}
\dot{v} \\
-\dot{u}
\end{array}\right)
$$

by applying to it the matrix

$$
g=-i \sigma_{3}=\left(\begin{array}{cc}
0 & -1 \\
1 & 0
\end{array}\right)
$$


This matrix also has the property

$$
g \sigma_{i} g^{-1}=-\left(\sigma_{i}\right)^{T}
$$

where the superscript $T$ means the transpose of the matrix. The transformation matrix applicable to the column vector of Eq.(B12) is $\dot{L}$ of Eq.(B9). Thus the matrix applicable to the row vector $(\dot{v},-\dot{u})$ in Eq. (B11) is

$$
\left\{g^{-1} \dot{L} g\right\}^{T}=g^{-1} \dot{L}^{T} g
$$

This is precisely the Hermitian conjugate of $L$.

In optics, this two-by-two matrix form appears as the coherency matrix, and it takes the form

$$
C=\left(\begin{array}{cc}
<E_{x}^{*} E_{x}> & <E_{y}^{*} E_{x}> \\
<E_{x}^{*} E_{y}> & <E_{y}^{*} E_{y}>
\end{array}\right),
$$

where $<E_{i}^{*} E_{j}>$ is the time average of $E_{i}^{*} E_{j}$. This matrix is convenient when we deal with light waves whose two transverse components are only partially coherent. In terms of the complex parameter $w$, the coherency matrix is proportional to

$$
C=\left(\begin{array}{cc}
1 & r e^{i \delta} \\
r e^{-i \delta} & r^{2}
\end{array}\right)
$$

if the $x$ and $y$ components are perfectly coherent with the phase difference of $\delta$. If they are totally incoherent, the off-diagonal elements vanish in the above matrix.

Let us now consider its transformation properties. As was noted by Opartrny and Perina [16], the matrix of Eq.(B16) is like

$$
C=\left(\begin{array}{cc}
t+z & x-i y \\
x+i y & t-z
\end{array}\right),
$$

where the set of variables $(x, y, z, t)$ is transformed like a four-vector under Lorentz transformations. Furthermore, it is known that the Lorentz transformation of this four-vector is achieved through the formula

$$
C^{\prime}=L C L^{\dagger}
$$


where the transformation matrix $L$ is that of Eq.(3.8). The construction of four-vectors from

the two-component spinors is not a trivial task [27,30]. The two-by-two representation of Eq.(B18) requires one more step of complication.

\section{APPENDIX C: TWO-DIMENSIONAL EUCLIDEAN GROUP}

Let us consider a two-dimensional plane and use the $x y$ coordinate system. Then $L_{z}$ defined as

$$
L_{z}=-i\left\{x \frac{\partial}{\partial y}-y \frac{\partial}{\partial x}\right\}
$$

will generate rotations around the origin. The translation generators are

$$
P_{x}=-i \frac{\partial}{\partial x}, \quad P_{y}=-i \frac{\partial}{\partial y}
$$

These generators satisfy the commutation relations:

$$
\left[L_{z}, P_{x}\right]=i P_{y}, \quad\left[L_{z}, P_{y}\right]=-i P_{x} \quad\left[P_{x}, P_{y}\right]=0
$$

These commutation relations are like those given in Eq.(5.3). They become identical if $L_{z}$, $P_{x}$ and $P_{y}$ are replaced by $J_{1}, N_{2}$ and $N_{3}$ respectively.

This group is not discussed often in physics, but is intimately related to our daily life. When we drive on the streets, we make translations and rotations, and thus make transformations of this $E(2)$ group. In addition, this group reproduces the internal internal

space-time symmetry of massless particles [1]. This aspect of the $E(2)$ group has been extensively discussed in the literature 26,27.33. 


\section{REFERENCES}

[1] E. Wigner, "On unitary representations of the inhomogeneous Lorentz group," Ann. Math. 40, 149-204 (1939).

[2] V. Bargmann, "Irreducible unitary representations of the Lorentz group," Ann. Math. 48, 568-640 (1947).

[3] P. A. M. Dirac, "Forms of relativistic dynamics," Rev. Mod. Phys. 21, 392-399 (1949).

[4] P. A. M. Dirac, "A remarkable representation of the $3+2$ de Sitter Group," J. Math. Phys. 4, 901-909 (1963).

[5] H. P. Yuen, "Two-photon coherent states of the radiation fields," Phys. Rev. A 13, 2226-2243 (1976).

[6] C. M. Caves and B. L. Schumaker, "New formalism for two-photon quantum optics. I. Quadrature phases and squeezed light," Phys. Rev. A 31, 3068-3092 (1985); B. L. Schumaker and C. M. Caves, "New formalism for two-photon quantum optics. II. Mathematical foundation and compact notation," Phys. Rev. A 31, 3093-3111 (1985).

[7] Y. S. Kim and M. E. Noz, Phase Space Picture of Quantum Mechanics (World Scientific, Singapore, 1991). pp 77-122.

[8] H. Bacry and M. Cadilhac, "Metaplectic group and Fourier optics," Phys. Rev. A 23, $2533-2536(1981)$.

[9] E. C. G. Sudarshan, R. Simon, and N. Makunda, "Paraxial-wave optics and relativistic front description. I. The scalar theory," Phys. Rev. A 28, 2921-2932 (1983); N. Makunda, R. Simon, and E. C. G. Sudarshan, "Paraxial-wave optics and relativistic front description. II. The vector theory," ibid. 28, 2933-2942 (1983).

[10] D. Han, Y. S. Kim, and M. E. Noz, "Wavelets, windows, and photons," Phys. Lett. A 206, 299-304 (1995). 
[11] P. Pellat-Finet and M. Buasset, "What is common to both polarization optics and relativistic kinematics?," Optik (Stuttgart) 90, 101-106 (1992).

[12] M. Gutierrez, J. C. Minano, C. Vega, and P. Benitez, "Application of Lorentz geometry to non-imaging optics: New three-dimensional ideal contractors," J. Opt. Soc. Am. A 13, 532-542 (1996).

[13] S. R. Cloude, "Group theory and polarization algebra," Optik 75, 26-36 (1986).

[14] R. Y. Chiao and T. F. Jordan, "Lorentz-group Berry phases in squeezed light," Phys. Lett. A 132, 77-81 (1988).

[15] M. Kitano and T. Yabuzaki, "Observation of Lorentz-group Berry phases in polarization optics," Phys. Lett. A 142, 321-325 (1989).

[16] T. Opartrny and J. Perina, "Non-image-forming polarization optical devices and Lorentz transformations - analogy," Phys. Lett. A 181, 199-202 (1993).

[17] D. Han, Y. S. Kim, and M. E. Noz, "Polarization optics and bilinear representation of the Lorentz group," Phys. Lett. A 219, 26-32 (1996).

[18] R. C. Jones, "New calculus for the treatment of optical systems," J. Opt. Soc. Am. 31, 488-493, (1941).

[19] W. Swindell, Polarized Light (Dowden, Hutchinson, and Ross, Inc., Stroudsburg, PA, 1975).

[20] F. L. Pedrotti and L. S. Pedrotti, Introduction to Optics, 2nd Ed. (Prentice Hall, Englewood Cliff, New Jersey, 1993). pp 280-297.

[21] D. Han, Y. S. Kim, and D. Son "E(2)-like little group for massless particles and polarization of neutrinos," Phys. Rev. D 26, 3717-3725 (1982).

[22] Y. S. Kim and L. Yeh, "E(2)-symmetric sheared states," J. Math. Phys. 33, 1237-1246 (1992). 
[23] A. S. Chirkin, A. A. Orlov, and D. Yu. Parashchuk, "Quantum theory of two-mode interactions in optically anisotropic media with cubic nonlinearities: Generation of quadrature- and polarization-squeezed light," Quantum Electron. 23, 870-874 (1993).

[24] R. Abraham and J. E. Marsden, Foundations of Mechanics, 2nd Ed.. (Benjamin/Cummings, Reading, MA, 1978).

[25] V. Guillemin and S. Sternberg, Symplectic Techniques in Physics (Cambridge University, Cambridge, 1984).

[26] Y. S. Kim and M. E. Noz, Theory and Applications of the Poincaré Group (Reidel, Dordrecht, 1986).

[27] D. Han, Y. S. Kim, and D. Son, "Photons, neutrinos, and gauge Transformations," Am. J. Phys. 54, 818-821 (1986).

[28] M. Born and E. Wolf, Principles of Optics, 6th Ed. (Pergamon, Oxford, 1980). pp 545-553.

[29] J. Perina, Coherence of Light (Van Nostrand Reinhold, London, 1971). pp 77-88.

[30] S. Baskal and Y. S. Kim, "Four-potentials and Maxwell field tensors from SL(2,C) spinors as infinite-momentum/zero-mass Limits of their massive counterparts," hep-th9512088 (1995).

[31] G. P. Parent and P. Roman, "On the matrix formulation of the theory of partial polarization in terms of observables," Nuovo Cimento 15, 370-388 (1960); R. Barakat, "Theory of the coherency matrix for light of arbitrary bandwidth," J. Opt. Soc. Am. 53, 317-323 (1963); H. Takenaka, "A unified formalism for polarization optics by using group theory," Nouvelle Revue d'Optique 4, 37-41 (1973).

[32] C. E. Baum and H. N. Kritikos, Electromagnetic Symmetry (Taylor and Francis, Washington, DC, 1995). 
[33] S. Weinberg, "Feynman rules for any spin II. massless particles." Phys. Rev. 134, B882896 (1964). 